\title{
Development of an Automated Urban Climate Monitoring System in Novi Sad (Serbia)
}

\author{
Ivan Šećerov ${ }^{A}$, Stevan Savić ${ }^{B}$, Dragan Milošević ${ }^{B}$, Vladimir Markovićc, Ivana Bajšanski ${ }^{D}$ \\ Received: August 24, 2015 | Revised: September 22, 2015 | Accepted: November 7, 2015
}

\begin{abstract}
This paper presents development and implementation of an automated urban climate monitoring system in mid-sized European city Novi Sad (Vojvodina, Serbia). The urban climate monitoring network contains 27 stations (25 in urban area and 2 in non-urbanized environment). To determine the representativeness station cites we used Stewart-Oke Local Climate Zones (LCZ) classification, authors' local knowledge and field surveys to delineate urban area and surrounding environments. For LCZ determination we used Lelovics-Gál automated GIS (Geographic Information System) method. Stations measure the values and every 10 minutes send the readings related to air temperature, relative humidity, battery voltage, status value and other technical information to the main server.

In order for the data stored in database server to be ready for usage in urban climate studies and for analyzing entire systems' work, we created Urban Path System tool (UP-SYS_tool) and URBAN-PATH Portal. Furthermore, the automatic data procession system visualizes the measured values from urban climate monitoring network in Novi Sad and informs the citizens about the most recent temperature, relative humidity and thermal comfort measurements.
\end{abstract}

These, in situ measurements should provide long-term time series in order to highlight the thermal differences among different urban structures in city/es (intra-urban and inter-urban comparisons), and contribute to better understanding of urban climate in the urban canopy layer.

Key words: urban climate, monitoring network, Local Climate Zones, UP-SYS_tool, URBAN-PATH Portal, Novi Sad, Serbia

\section{Introduction}

According to the most urban climate studies, air temperature in the urban canopy layer shows the most obvious modification compared to rural areas. This urban warming is referred to as the urban heat island (UHI) and its magnitude is the UHI intensity $\left(\Delta \mathrm{T}_{\mathrm{u}-\mathrm{r}}\right)$ (Oke, 1987; Unger, et al., 2013).
In order to facilitate consistent and climatologically relevant classifications of urban and rural field sites for temperature observations at standard screen height, Stewart and Oke (2012) developed the Local Climate Zone (LCZ) classification system. It contains 1o built and 7 land cover types and it has intended to be used for the whole of Earth's surface. This LCZ system is based on the earlier scientific works (Auer, 1978; Ellef-

\footnotetext{
A Department of Geography, Tourism and Hotel Management, Faculty of Sciences, University of Novi Sad, Trg Dositeja Obradovića 3, 21000 Novi Sad, Serbia

B Climatology and Hydrology Research Centre, Faculty of Sciences, University of Novi Sad, Trg Dositeja Obradovića 3, 21000 Novi Sad, Serbia

c Center for Spatial Information of Vojvodina, Faculty of Sciences, University of Novi Sad, Trg Dositeja Obradovića 3, 21000 Novi Sad, Serbia

D Department of Architecture and Urban Planning, Faculty of Technical Sciences, University of Novi Sad, Trg Dositeja Obradovića 6, 21000 Novi Sad

* Corresponding author: Stevan Savić, e-mail: stevan.savic@dgt.uns.ac.rs
} 
sen, 1991; Oke, 2004; Oke, 2009; Stewart, 2011) and extensive field surveys. Furthermore, the LCZ classification system provides a methodological background to objectively compare the thermal characteristics of different areas within a city (intra-urban observations) (Milošević et al., 2015) and its surroundings as well as between cities (inter-urban observations) (Lelovics, et al., 2014; Stewart, et al., 2014).

During the years of 2013 and 2014 there were activities related with the development of urban monitoring and online information systems regarding spatial distribution of air temperature, relative humidity and human thermal comfort conditions in mid-sized cities of Szeged (Hungary) and Novi Sad (Serbia). Within the framework of the EU-funded project (URBAN-PATH; http://en.urban-path.hu), climatologists from University of Szeged and University of Novi Sad installed two urban climate monitoring networks (24 stations in Szeged and 27 stations in Novi Sad) in order to obtain longterm and effective measurement data. The networks aim to highlight the differences between thermal characteristics of the neighborhoods and cities (intra-urban and inter-urban comparisons) and their temporal resolution allowing the exploration of both the diurnal as well as the seasonal peculiarities. Knowledge based on the developed systems, should contribute to the effectiveness of sustainable development and climate-conscious urban planning strategies, mitigate the impacts of global climate change and maintain the health of the urban population (Savić, et al., 2013; Savić, et al., 2014; Unger, et al., 2014). Up to now, in scientific journals there are papers related to automatic monitoring networks set up in the urban canopy layer and the detection of patterns of human comfort conditions (Watkins, et al., 2002; Mikami, et al., 2003; Dabberdt, et al., 2005; Chang, et al., 2010; Schroeder, et al., 2010; Basara, et al., 2011; Davies, et al., 2011; Houet, Pigeon, 2011; Hung, Wo, 2012; Young, at al., 2012; Petralli, et al., 2013; HiTEmp Project, 2014; Castell, et. al., 2014; Fenner, et al., 2014, Chapman, et al., 2014; Unger, et al., 2015a; 2015b).

The main goal of this study is to introduce the urban climate monitoring network and public information system recently implemented in Novi Sad. This research provides a new approach of LCZ mapping and selecting station cites based on an automated Geographic Information System method (Lelovics, et al., 2014). Furthermore, this paper explains the methods of data representation and communication between stations and servers, working with data stored in database and real-time public availability of the data.

\section{Investigated area}

Novi Sad is a mid-sized European city in Vojvodina, the northern Province of the Republic of Serbia (Figure

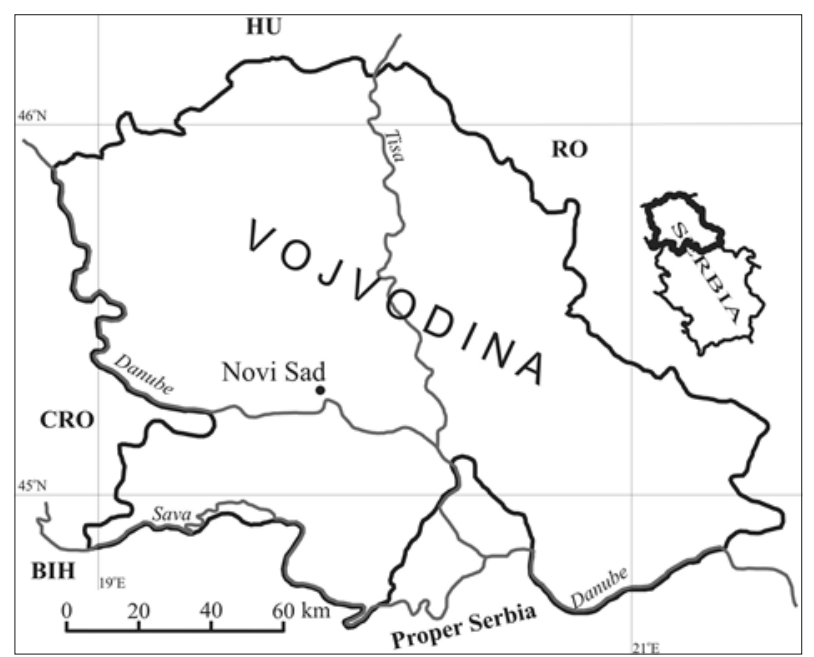

Figure 1. Location of Novi Sad in Autonomous Province of Vojvodina and its location in Republic of Serbia

1), located on a plain between 80 and $86 \mathrm{~m}$ a.s.l. Hence, the climate is generally free of orographic effects. Based on a population of 340.000 (data from 2014), Novi Sad is the second largest metropolitan region in Serbia with built-up area of $112 \mathrm{~km}^{2}$. The Danube River passes through the southern and eastern edges of the urban area; its width varies from 260 to $680 \mathrm{~m}$. The relatively narrow Danube-Tisza-Danube Canal passes through the northern part of the city. The northern slopes of the Fruška Gora Mountains (which have a maximum peak of $538 \mathrm{~m}$ a.s.l.) are located south of the Novi Sad urban area (Unger at al., 2011).

Novi Sad region has a Cfb climate (temperate climate, fully humid, and warm summers, with at least four $\mathrm{T}_{\text {mon }} \geq+10^{\circ} \mathrm{C}$ ) according to Köppen-Geiger climate classification (Kottek, et al., 2006). The mean monthly air temperature ranges from $-0.4{ }^{\circ} \mathrm{C}$ in January to $21.7^{\circ} \mathrm{C}$ in July. The mean annual precipitation is $598 \mathrm{~mm}$ (based on data registered from 1949 to 2013).

\section{Methods for defining Local Climate Zones and station sites}

For defining and delineating the LCZ types in Novi Sad built-up area, the automated Geographic Information System (GIS) method developed by Lelovics, et al. (2014) was used (Figure 2). The study area has been divided into lot area polygons (Gál and Unger, 2009), which consists of a building and the area of influence around it, as basic areas in the calculation of surface parameters necessary to characterize the LCZ types (totally 47.000 polygons). We used seven out of ten physical properties identified and designated for LCZ classification by Stewart and Oke (2012) (Figure 2). The aspect ratio, surface admittance and anthropogenic heat output were omitted because of the lack of data or inappropriate type of urbanization. The calcu- 


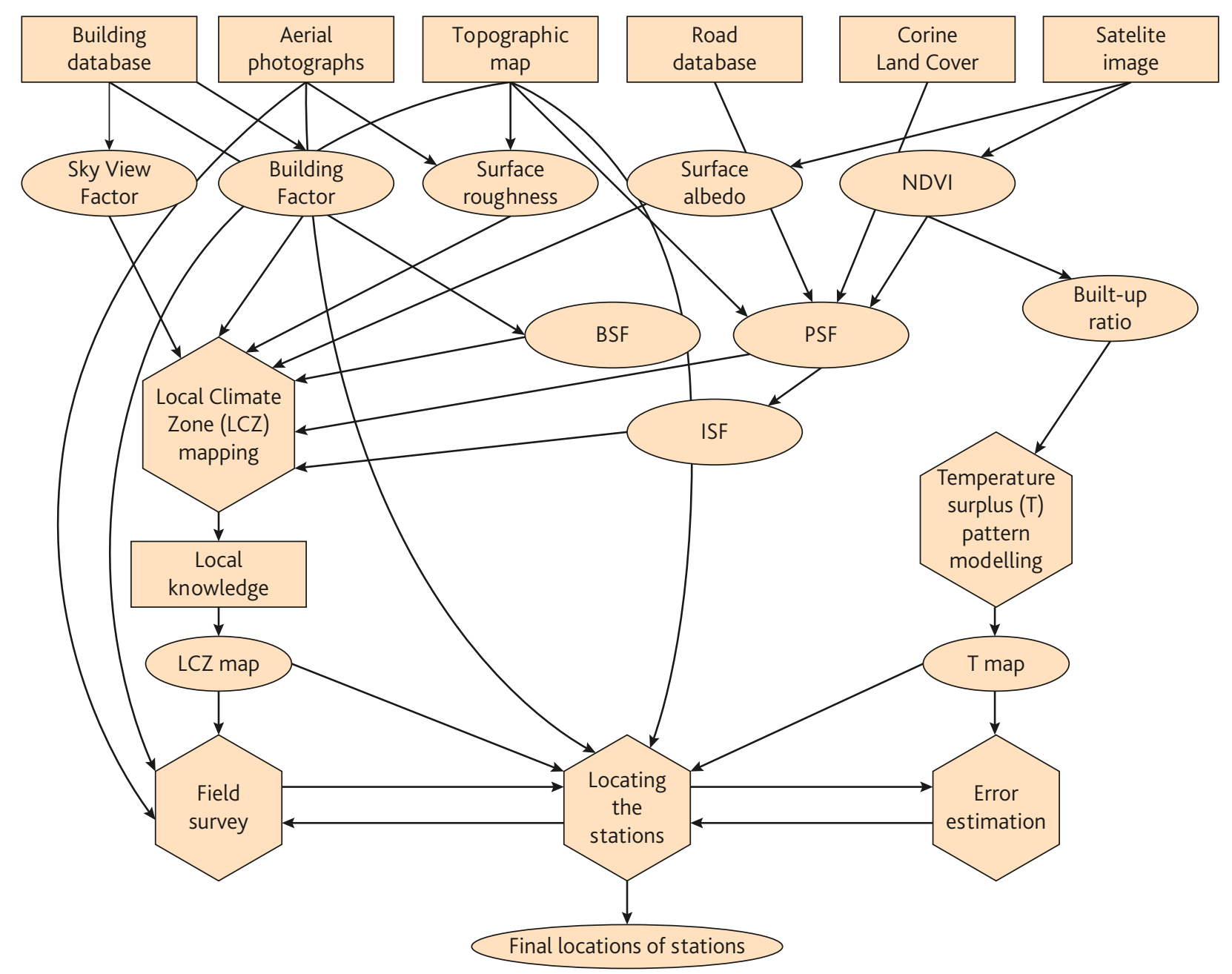

Figure 2. Process of identifying and delineating LCZs and selecting the representative station sites for urban monitoring network in Novi Sad, Serbia (based on Lelovics et al., 2014). Note: NDVI - Normalized Difference Vegetation Index, BSF Building Surface Fraction, PSF - Pervious Surface Fraction, ISF - Impervious Surface Fraction

lation processes of the physical properties and the utilized database are presented in Lelovics, et al. (2014).

Due to the urbanization characteristics of Novi Sad and complemented by the authors' local knowledge of the study area, the 7 LCZ built classes are detected and delineated. Figure 3 shows the spatial pattern of these $7 \mathrm{LCZ}$ classes within the city, named as: $\mathrm{LCZ}_{2}$ - Compact mid-rise, $\mathrm{LCZ}_{3}$ - Compact low-rise, LCZ 5 - Open mid-rise, LCZ 6 - Open low-rise, LCZ 8 - Large low-rise, LCZ 9 - Sparsely built and LCZ 10 - Heavy industry. The types and areal distribution of these zones within the urban area of Novi Sad supplemented by two land cover types (A - Dense trees, D Low plants) are listed in Table 1.

In order to have a representative urban monitoring network in Novi Sad the locations of all stations were based on the four criteria (Lelovics, et al., 2014): 1) the sites had to be surrounded by at least $250 \mathrm{~m}$ wide homogeneous LCZ areas, and the number of stations per each LCZ had to be approximately proportional to the areas of different LCZs; 2) the site's representativeness in terms of its microenvironment, i.e. the selected site had to be typical to the LCZ where the station was located; 3) the sites had to be located near the areas where high and low temperature surpluses occurred, as well as near local maxima and around spatial tem-

Table 1. Spatial characteristics of LCZ built types and distribution of stations in each LCZs

\begin{tabular}{|c|c|c|c|}
\hline $\begin{array}{c}\text { LCZ built } \\
\text { types }\end{array}$ & $\begin{array}{c}\text { Number of } \\
\text { patches }\end{array}$ & $\begin{array}{c}\text { LCZ proportion } \\
\text { in } \%\end{array}$ & $\begin{array}{c}\text { Number of } \\
\text { stations }\end{array}$ \\
\hline 2 & 6 & 9.6 & 3 \\
\hline 3 & 5 & 5.2 & 2 \\
\hline 5 & 8 & 15.1 & 6 \\
\hline 6 & 19 & 42.3 & 9 \\
\hline 8 & 6 & 10.1 & 1 \\
\hline 9 & 5 & 13.0 & 3 \\
\hline 10 & 4 & 4.7 & 1 \\
\hline A & & & 1 \\
\hline$D$ & \multicolumn{2}{r|}{} \\
\hline
\end{tabular}




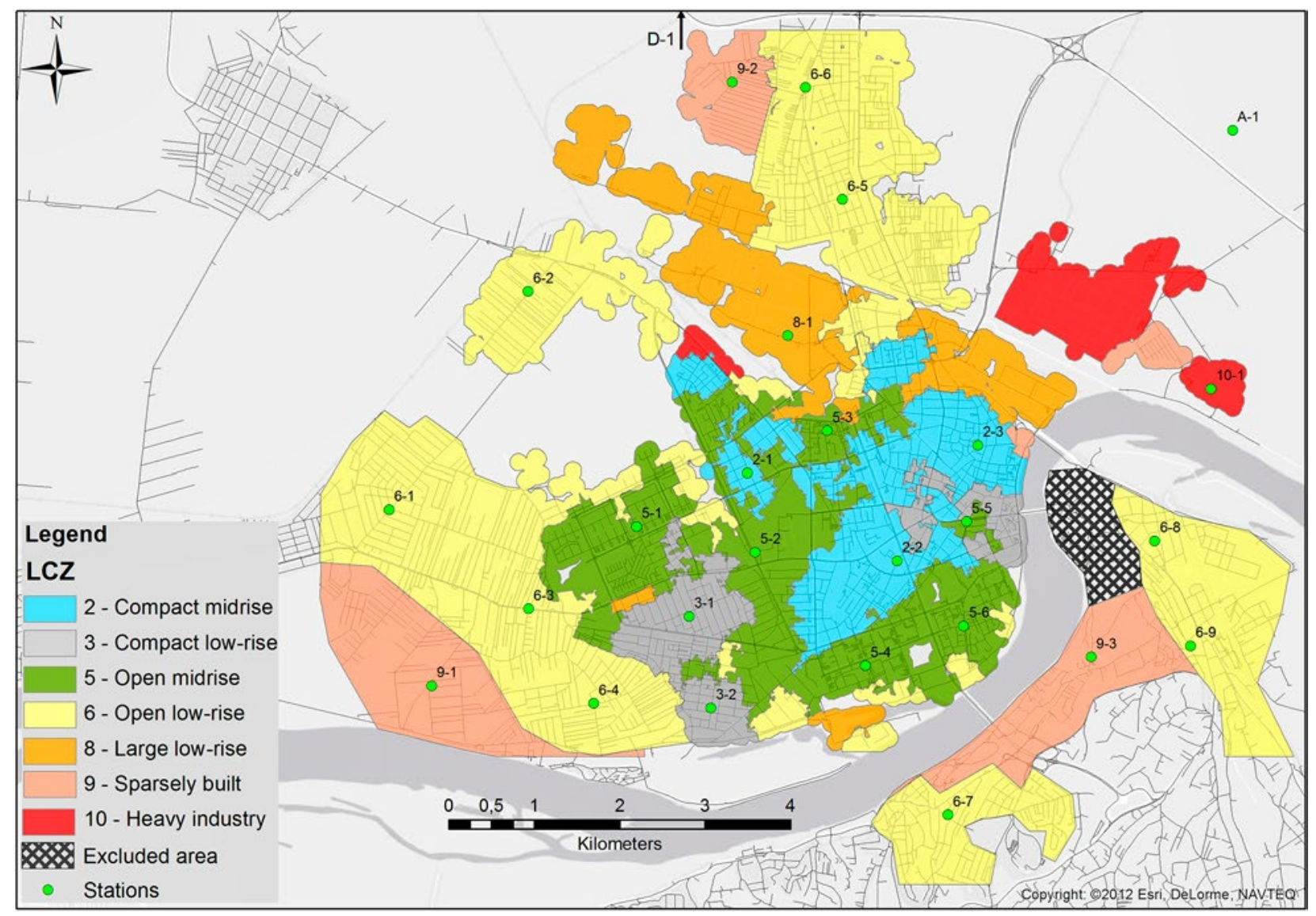

Figure 3. The obtained LCZ built classes and station locations of the urban monitoring network in Novi Sad (Serbia). Note (Station labels): first character - LCZ type; second character - station number in the given LCZ type

perature stretches, as indicated by the modeled temperature pattern (Unger et al., 2011); 4) the site's suitability for instrument mounting (for instance, safety, constant electricity supply, stability of lamppost).

The urban monitoring network in Novi Sad contains 25 station sites staggered at 7 different LCZ classes (Figure 3). Two more stations are located in the land cover type LCZ classes in the north (D-1) and northeast (A-1) to the urban zone, respectively, and they represent general climate conditions in the non-urbanized areas. The distribution of stations per LCZs is shown in Table 1.

\section{Technical background of the urban climate network}

All mounted stations are equipped with ChipCap 2 sensors in radiation protection screens with dimensions of $200 \times 240 \mathrm{~mm}$. Fully calibrated temperature and humidity sensors are developed by the General Electric Measurement \& Control Company. The accuracy of the air temperature sensor is $\pm 0.3^{\circ} \mathrm{C}$ and of the relative humidity sensor is $\pm 2 \%(20 \sim 80 \% \mathrm{RH})$. The sensors and all equipment were installed at least $4 \mathrm{~m}$ above the ground (with exceptions $\pm 0.2 \mathrm{~m}$ ) on arms (50 cm long) fixed to selected lampposts (Figure 4).

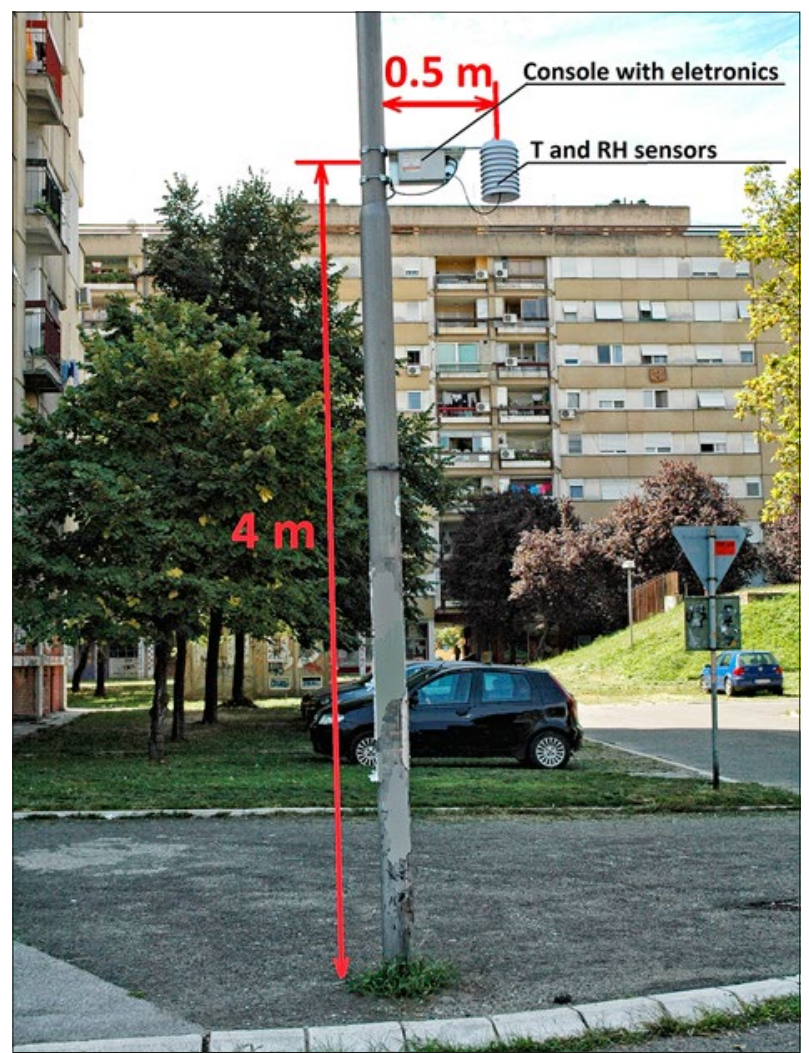

Figure 4. Example of monitoring network station in Novi Sad (Serbia) mounted on a lamppost 
Two stations in non-urbanized areas were installed at $2 \mathrm{~m}$ above the ground.

Central processor, EPROM chip as data storage, GPRS/EDGE/3G modem, battery and charger are settled in the station console. Only one station (A-1, Figure 3) has continuous power supply, but all other stations have power supply only when the city lights are on. During the day, i.e. when the city lights are off, stations work on battery supply. The stations can operate up to 10-15 days using battery power alone. The system time of the stations is in UTC and this time has regularly synchronized by the main server (installed at University of Novi Sad, Faculty of Sciences). Stations measure the values every minute and every $10 \mathrm{~min}$ utes send the readings related to air temperature, relative humidity, battery voltage, status value and other technical information to the main server. In case of problems due to mobile Internet provider or if the main server does not receive data due to local network problems, or problems of any kind, the station resends the data (using built in algorithm) until it is delivered to the main server. All files which could not be delivered to the main server at the proper time are stored in the station's internal memory (maximum 15 months or approximate 700.000 files) and can be accessed later manually using serial connection, i.e. stations are programmed not to lose the measured data.

\section{Data representation and communication between stations and servers}

All stations temperature and humidity measurements are included in files (with extension .txt), together with other statistics related to stations work (Figure 5). File naming convention and structure is defined in such manner that it is always possible to determine in short time what is stored in file and from which station. Each file name begins with string defining station ID (two/three characters joined with '-' and station number). Delimiter that separates ID from other information is character ', ', and in the forward goes time representation. Two digits represent the year, joined with two digits that represent month and two final digits represent day. Again delimiter ' ' is used to distinguish date from time, which is used as $24 \mathrm{~h}$ format. Two digits are used to represent hour and similary two digits are used to represent minutes. On the other hand, seconds are not stored, but two trailing zeros are left for future implementations. Based on value of the Transmission Period - TP, two digits representing minutes are generated based on TP value as:

a) if $\mathrm{TP}=1$, two digits represent file creation time

b) if $\mathrm{TP}>1$, two digits represent time of last created file.
Files are meant to be human readable and stored data must contain all measurements together with statistics about station work. Because of time needed to send file to the server, size of file must be as small as possible. Costs concerned Internet mobile provider may vary so keeping files as small as possible are desired.

File structure is defined in such way that each value begins with new line. Characters as space, ASCII code ox20, tab, ASCII code oxy must not be included. Data is represented using variables and they are represented with UNIX EOL (Unix end of line) - LF (line feed, ASCII code oxoA). First two/three characters in each row represent variable names and they are defined with capital letters. Delimiter between variable and stored data is defined as '?' (ASCII code ox3A). If stored data must contain letter it is stored as small letter.

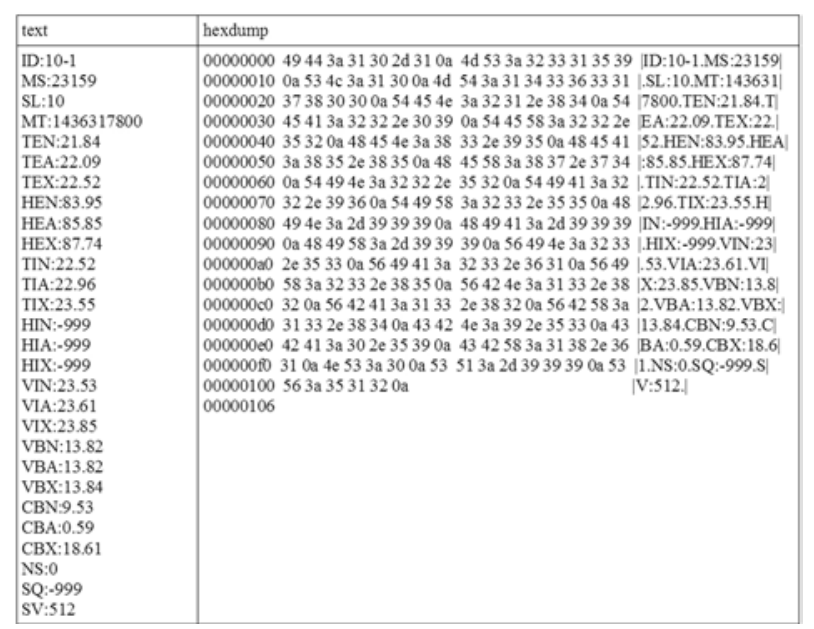

Figure 5. The file naming convention and file structure. Example for file: 10-1_150708_011000.txt

After transmission time is reached (10 minutes interval), station connects to primary server using FTP (file transfer protocol). FTP is a protocol build for file transfer, proven to be reliable and is used for past 30 years. It supports authentication and can be used in combination with some crypto protocols such as SSL (Secure Socket Layer) to provide good layer of security. If primary server is not available due to network errors, retransmission will be attempted defined number of times, after which file will be sent to secondary server. When FTP session is established, file(s) containing data are sent to the server, after which dynamic configuration of station will be performed. Each station will search for its configuration file ("_get" file). If there is difference between configuration file and current station configuration, station will store applied configuration to its "_set" file located on server. During each FTP session, time synchronization is performed. File „time.conf“ containing current time 


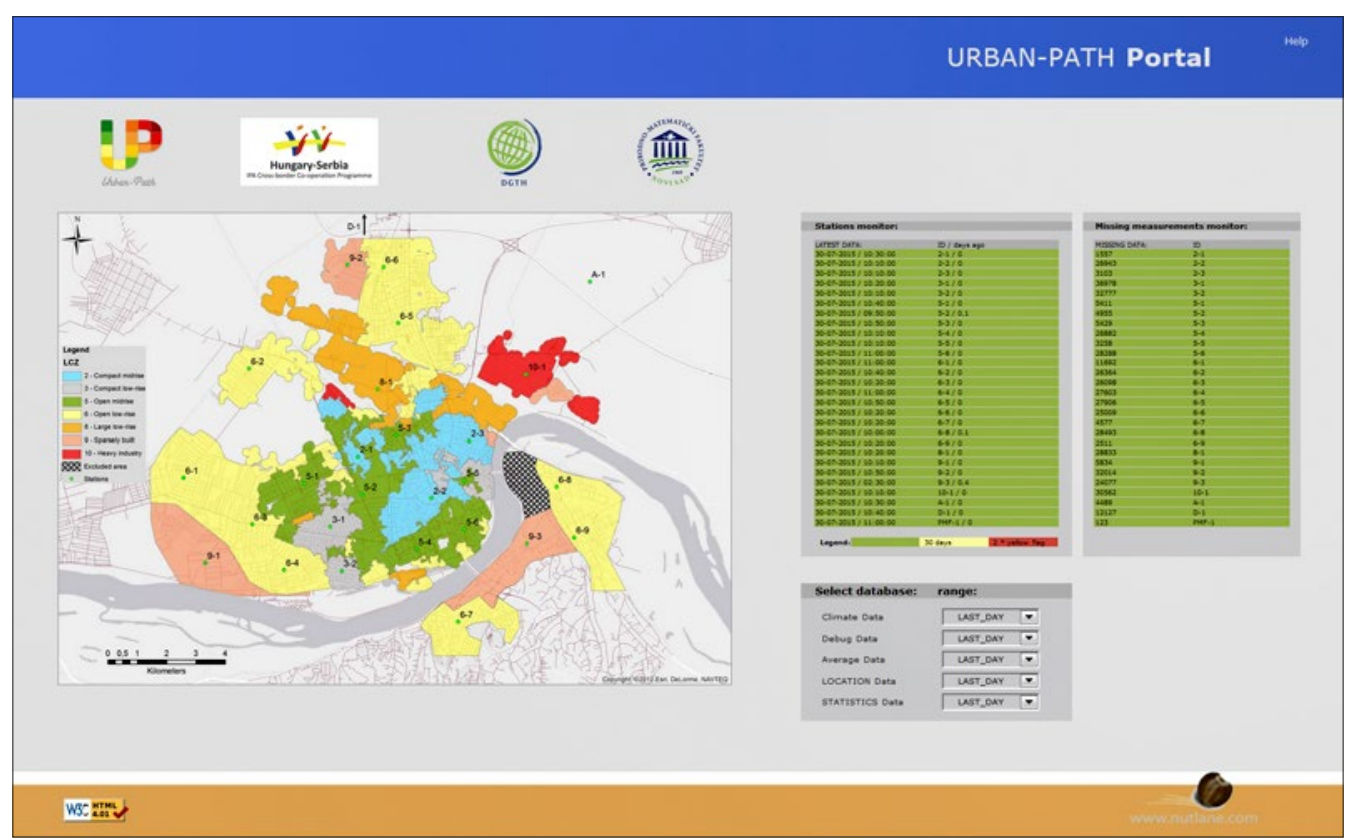

Figure 6. URBAN-PATH Portal main page with data monitoring and database selection

using Unix time format is generated per minute basis by server.

Data uploaded to the primary server are being processed by UP-SYS_tool (Urban Path System tool). Each data is being processed whether it contains climatological measurement or debug data with statistics about station work. Error detection and notification is performed through entire UP-SYS_tool work. As final result of UP-SYS_tool work, data are stored into database server. Using defined periods, UP-SYS_tool starts archiving process to relocate and compress processed files to archive location. After that they can be used, if needed, for recovery.

\section{Working with stored data}

Data stored in database server are ready to be used in urban climate studies and for analyzing entire systems' work. URBAN-PATH Portal is application built to support all that demands (Figure 6). It can be used for two purposes:

a) to get data from database in desired format and

b) to monitor current system status.

Latest inserted data for each station (stations monitor) is shown together with its 'age'. If last inserted data is older then defined thresholds, line containing it is colored with different warning color. Next to stations monitor is missing measurements monitor, used to provide adequate information about number of missing data per station. Although system is built to support different levels of problems whether they are hardware or software or related to connection problems, final result is - data not being stored into data- base server. UP-SYS_tool periodically check database for missing data and stores results into separate database, which is used for missing measurements monitor.

In order to work with data, whether they are classified as measurement or debug, proper database must be selected. After database selection, each table containing station data (except for LOCATION data, which is global information about stations location, LCZ, etc.) is shown together with its minimum and maximum inserted values. Time period for data selection can be fine-tuned using two methods of selection: Unix time or time/date form. Selection can be done using some or all stations tables, and for each station different columns representing separate data can be selected. Whatever selection is given, URBAN-PATH Portal will provide properly formatted queries to database server and provide user with desired data. Data can be output on screen, or can be stored into file to import into third party programs for further analyze.

\section{Public display system}

The visualization of the measured values (either as graphs or as maps) from urban climate monitoring network in Novi Sad was provided from automatic data procession system. This system is developed and installed at Department of Climatology and Landscape Ecology in University of Szeged.

Every 10 minutes, the data from monitoring network, stored into the main server at Faculty of Science, University of Novi Sad, transmitted to the main server at University of Szeged and the automatic data procession system creates the final two (site and spatial) databases (Figure 7) in order to present these data as 
charts and maps on the public homepage of the URBAN-PATH project (http://en.urban-path.hu/monitoring-system.html). All of the measured and calculated values can be accessed a way that the time of the maps and charts can be freely modified by the visitors (Savić and Unger, 2014; Unger et al., 2015b). Additionally, public display is installed at frequently visited place, i.e. in the main building of the University of Novi Sad.

The received data from the monitoring network are stored in one text file per day on the server, and also stored in a MySQL database. Every 10 minutes Java software calculates the Physiologically Equivalent Temperature (PET) value (Mayer, Höppe 1987) describing the human comfort conditions for each station using the temperature and relative humidity values measured, as well as global radiation and wind speed data using WRF model predictions (Figure 8). The results of these calculations are also stored in the MySQL database (Figure 7) (Savić, Unger, 2014; Unger, et al., 2015b).

For the automatic interpolation of the spatial patterns of the measured and calculated data Java software was developed. This program applies simple linear interpolation for a $500 \mathrm{~m}$ resolution grid of the study area using the data of the three nearest stations of each grid point. In order to avoid the incorrect interpolation in the edge of the study area, the two rural stations considered as the background station, thus at the bordering (non-urban) grid points we used the data of the nearest rural station, and all of these points were added to the original measurement points for the interpolation (Figure 7). The coordinates of the grid points and the stations are in the Unified Hun- garian Projection, but at the end of the interpolation they were converted to WGS84 latitude and longitude coordinates because it is more appropriate for the further processing (drawing maps with GrADS, comparing the measurements with weather prediction models). At the first hand we applied a weighting constant (currently it is 1) in the interpolation and after further investigations we will alter this constant using the statistical connection between the surface parameters (e.g. built up ratio, SVF, green area, water surface) and the measured temperature, relative humidity or the PET in order to increase the precision of the interpolation (Figure 7). The final patterns are stored in another, the spatial database, which is technically a NetCDF file. The public project homepage presents these patterns as maps created by GrADS and PHP scripts (Savić, Unger, 2014; Unger, et al., 2015b).

\section{Conclusions}

There is a huge number of papers that deal with urban climate issues. On the other hand, in situ long-term measurements based on automatic weather station monitoring network is still rare in cities. The use of automatic weather stations is the most suited method for understanding the spatial and temporal characteristics of the urban climate and in order to assist in formulating adaptation and mitigation strategies to meet the challenges of climate change. Within the frame of the URBAN-PATH project, the urban climate monitoring system in Novi Sad (Serbia) has developed to visualize the observed air temperature and relative humidity data along with calculated human comfort index. The total of 27 stations were installed within

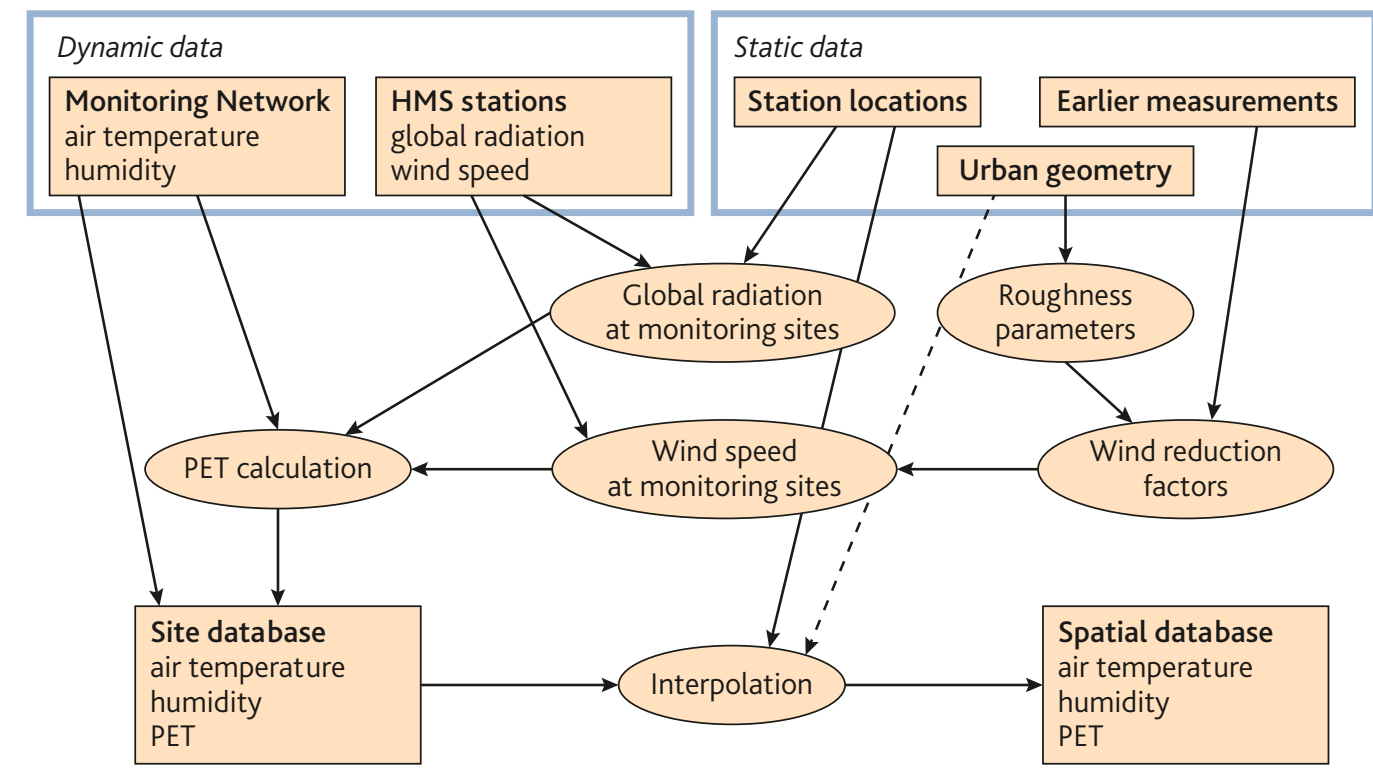

Figure 7. Flow chart of the automatic data processing of the monitoring system (Savić and Unger, 2014; Unger et al., 2015b) 


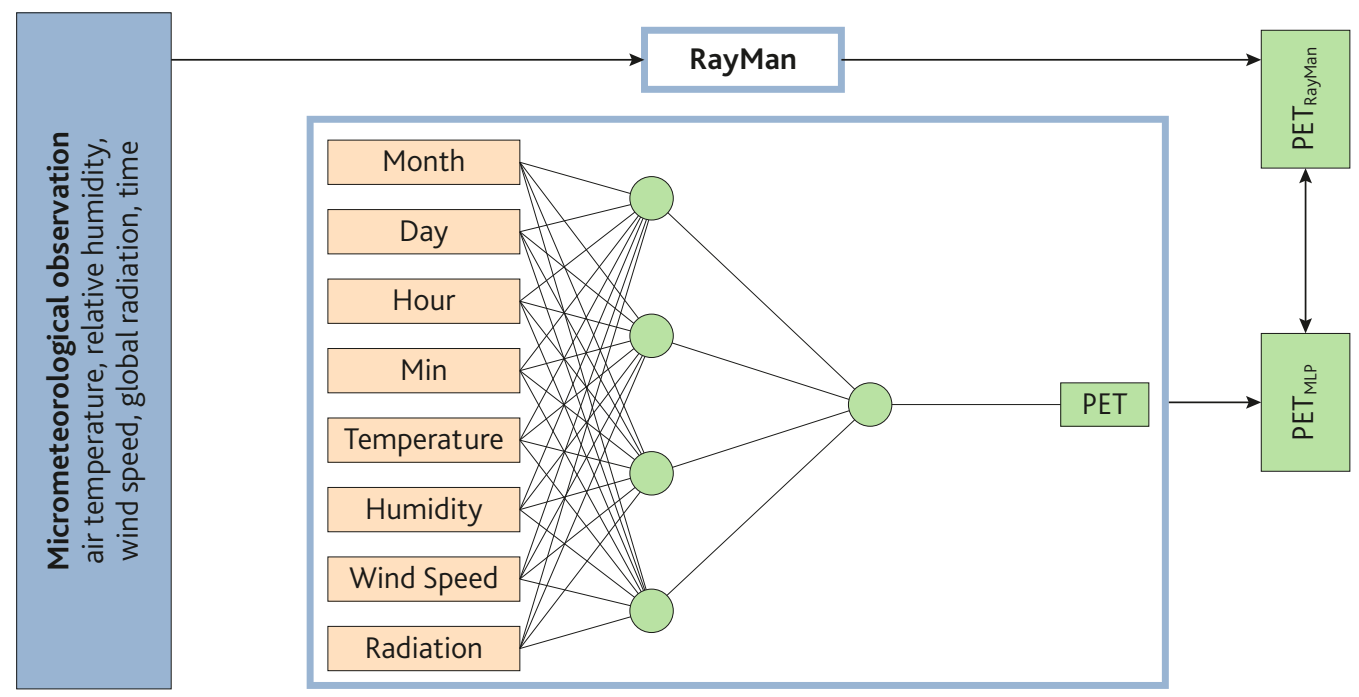

Figure 8. Flow chart of the development of the Multilayer Perception (MLP) network structure (Savić and Unger, 2014; Unger et al., 2015b)

the seven delineated LCZs built classes and two land cover types. Defined locations and station' distribution per each LCZ is based on the determined criteria, complemented by the authors' local knowledge and field surveys.

Monitoring system is using well-defined files that can send number of information in short time with minimal transfer costs to mobile provider. Stations configuration can be performed without physical access or any sophisticated software (configurations are stored as plain text files). Proposed file structure can be used for other data collection, which have demands for frequent measurements. Initial demands from this system were to support and process data for $27 \mathrm{sta}-$ tions. During first year, one more station with different type of measurement was integrated into system, and different types of stations can easily be integrated into the system as well. Tests showed that UP-SYS tool could process up to 2000 stations in less than six minutes if it is performed on moderate cost hardware. Being BASH script (Bourne Again Shell), UP-SYS tool can be easily altered to fit different needs and can be used on different platforms. Although script has proved excellent working performance with large number of files, sophisticated programming language such as $\mathrm{C}$ would improve it work in many ways.

Finally, it can be stated that the urban climate monitoring system installed in Novi Sad is adequate to a) provide a long-term data for further research in urban heat island and thermal comfort issues and b) inform the citizens about the most recent temperature, relative humidity and thermal comfort measurements. The planned operation time of this system will be minimum 5 years. Data series from urban climate monitoring network will allow for more detailed and versatile intra-urban and inter-urban comparisons and help local authorities in creating environmental and health mitigation strategies.

\section{Aknowledgements}

Development of the urban climate monitoring network in Novi Sad was partly supported by the Hungarian-Serbian IPA Cross-border Co-operation Programme through the URBAN-PATH project (no. HUSRB/1203/122/166). This study was partly supported as a project funded by the Serbian Ministry of Education, Science and Technological Development 176020 .

\section{References}

Auer, A. H. 1978. Correlation of land use and cover with meteorological anomalies. Journal of Applied Meteorology and Climatology 17, 636-643. doi: http:// dx.doi.org/10.1175/1520-0450(1978)017<0636:COLU $\mathrm{AC}>2.0 . \mathrm{CO} ; 2$

Basara, J. B., Illston, B. G., Fiebrich, C. A., Browder, P. D., Morgan, C. R., McCombs, A., Bostic, J. P., McPherson, R. A., Schroeder, A. J., Crawford, K. C. 2011. The Oklahoma City Micronet. Meteorological Applications 18, 252-261. doi:10.1002/met.189

Castell, N., Kobernus, M., Liu, H.Y., Schneider, P., Lahoz, W., Berre, A. J., Noll, J. 2014. Mobile technologies and services for environmental monitoring: The Citi-Sence-MOB approach. Urban Climate, in press. http://dx.doi.org/10.1016/j.uclim.2014.08.002

Chang, B., Wang, H. Y., Peng, T. Y., Hsu, Y. S. 2010. Development and evaluation of a city-wide wireless weather sensor network. Journal of Education Technology \& Society 13, 270-280. ERIC number: EJ899892 
Chapman, L., Muller, C.L., Young, D. T., Warren, E. L., Grimmond, C. S. B., Cai, X-M., Ferranti, E. J. S. 2014. The Birmingham Urban Climate Laboratory: An open meteorological testbed and challenges of the smart city. Bulletin of the American Meteorological Society. doi: 10.1175/BAMS-D-13-00193.1

Dabberdt, W., Koistinen, J., Poutiainen, J., Saltikoff, E., Turtiainen, H. 2005. The Helsinki mesoscale testbed - An invitation to use a new 3-D observation network. Bulletin of the American Meteorological Society 86, 906-907. doi:10.1175/BAMS-86-7-906

Davies, L., Bell, J. N. B., Bone, J., Head, M., Hill, L., Howard, C., Hobbs, S. J., Jones, D. T., Power, S.A., Rose, N., Ryder, C., Seed, L., Stevens, G., Toumi, R., Voulvoulis, N., White, P. C. L. 2011. Open air laboratories (OPAL): a community-driven research programme. Environmental Pollution 159, 2203-2210. doi:10.1016/j.envpol.2011.02.053

Fenner, D., Meier, F., Scherer, D., Polze, A. 2014. Spatial and temporal air temperature variability in Berlin, Germany during the years 2001-2010. Urban Climate 10, 308-331. http://dx.doi.org/10.1016/j. uclim.2014.02.004

Gál, T., Unger, J. 2009. Detection of ventilation paths using high-resolution roughness parameter mapping in a large urban area. Building and Environment 44,198-206. doi:10.1016/j.buildenv.2008.02.008

HiTemp Project 2014. High Density Measurements within the Urban Environment. http://www.birmingham.ac.uk/schools/gees/centres/bucl/hitemp/ index.aspx

Houet, T., Pigeon, G. 2011. Mapping urban climate zones and quantifying climate behaviors - An application on Toulouse urban area (France). Environmental Pollution 159, 2180-2192. doi:10.1016/j.envpol.2010.12.027

Hung, T. K., Wo, O. C. 2012. Development of a community weather information network (Co-WIN) in Hong Kong. Weather 67, 48-50. doi:10.1002/wea.1883

Ellefsen, R. 1991. Mapping and measuring buildings in the canopy boundary layer in ten U.S. cities. Energy and Buildings 15-16, 1025-1049. doi:10.1016/03787788(91)90097-M

Kottek, M., Grieser, J., Beck, C., Rudolf, B., Rubel, F. 2006. World map of the Köppen-Geiger climate classification updated. Meteorologische Zeitschrift 15, 259-263. doi:10.1127/0941-2948/2006/0130

Lelovics, E., Unger, J., Gál, T., Gál, C. V. 2014. Design of an urban monitoring network based on Local Climate Zone mapping and temperature pattern modeling. Climate Research 60, 51-62. doi:10.3354/ cro122O

Mayer, H., Höppe, P. 1987. Thermal comfort of man in different urban environments. Theoretical and Applied Climatology 38, 43-49. doi:10.1007/BFoo866252
Mikami, T., Ando, H., Morishima, W., Izumi, T., Shioda, T. 2003. A new urban heat island monitoring system in Tokyo. 5th International Conference on Urban Climate, Lodz, Poland, O.3.5.

Milošević, D., Savić, S., Unger, J., Gál, T. 2015. Urban climate monitoring system suitability for intraurban thermal comfort observations in Novi Sad (Serbia) - with 2014 examples. ICUC9 - 9th International Conference on Urban Climate jointly with 12th Symposium on the Urban Environment, 2oth24th July 2015, Toulouse, France, Extended Abstracts: $6 \mathrm{pp}$.

Oke, T. R. 1987. Boundary Layer Climates (2nd ed.). Routledge, London-New York

Oke, T. R. 2004. Initial Guidance to Obtain Representative meteorological Observations at Urban Sites. IOM Report 81, WMO/TD no. 1250, Geneva, 51 pp.

Oke, T. R. 2009. The need to establish protocols in urban heat island work. Symposium \& Eighth Symposium on Urban Environment, 11-15 January, Phoenix

Petralli, M., Masetti, L., Brandani, G., Orlandini, S. 2013. Urban planning indicators: useful tools to measure the effect of urbanization and vegetation on summer air temperatures. International Journal of Climatology 34, 1236-1244. doi:10.1002/joc.3760

Savić, S., Milošević, D., Lazić, L., Marković, V., Arsenović, D., Pavić, D. 2013. Classifying Urban Meteorological Stations Sites by "Local Climate Zones": preliminary results for the City of Novi Sad (Serbia). Geographica Pannonica 17, 60-68.

Savić, S., Unger, J., Milošević, D., Lelovics, E., Gál, T. 2014. Mapping of local climate zones in two neighboring Central-European city located in similar geographical environments. IGU regional conference - Changes, Challenges, Responsibility, Kraków, Poland, IGU 2014 Book of Abstracts, IGU2014-0003

Savić, S., Unger, J. 2014. Evaluation and public display of urban patterns of human thermal conditions (URBAN-PATH project). Urban Climate News 51, 12-18 (link: http://urban-climate.org/newsletters/ IAUC054.pdf)

Schroeder, A. J., Basara, J. B., Illstron, B. G. 2010. Challenges Associated with Classifying Urban Meteorological Stations: The Oklahoma City Micronet Example. The Open Atmospheric Science Journal 4, 88-100

Stewart, I. D. 2011. A systematic review and scientific critique of methodology in modern urban heat island literature. International Journal of Climatology 31, 200-217. doi:10.1002/joc.2141

Stewart, I. D., Oke, T. R. 2012. Local Climate Zones for urban temperature studies. Bulletin of the American Meteorological Society 93, 1879-1900. doi:10.1175/ BAMS-D-11-0oo19.1 
Stewart, I. D., Oke, T. R., Krayenhoff, E. S. 2014. Evaluation of the 'local climate zone' scheme using temperature observations and model simulations. International Journal of Climatology 34, 1062-1080. doi:10.1002/joc.3746

Unger, J., Savić, S., Gál, T. 2011. Modelling of the annual mean urban heat island pattern for planning of representative urban climate station network. Advances in Meteorology 2011, 9 pages. doi:10.1155/2011/398613

Unger, J., Lelovics, E., Gál, T. 2013. A vector-based GIS method for mapping of local climate zones and its application in a Central-European city. Proceedings of the International Conference on Urban Climate and History of Meteorology, 25th/26th February 2013 , Firenze, Italy, 244-253

Unger, J., Savić, S., Gál, T., Milošević, D. 2014. Urban climate and monitoring network system in Central European cities. University of Novi Sad, Faculty of Science - University of Szeged, Department of Climatology and Landscape Ecology, Novi Sad - Szeged, $101 \mathrm{pp}$.
Unger, J., Savić, S., Gál, T., Milošević, D., Marković, V., Gulyás, Á., Arsenović, D. 2015a. Urban climate monitoring networks based on LCZ concept. ICUC9 - 9th International Conference on Urban Climate jointly with 12th Symposium on the Urban Environment, 20th-24th July 2015, Toulouse, France, Extended Abstracts: 6 pp.

Unger, J., Gál, T., Csépe, Z., Lelovics, E. Gulyás, Á. 2015b. Development, data processing and preliminary results of an urban human comfort monitoring and information system. Idójárás (in press)

Watkins, R., Palmer, J., Kolokotroni, M., Littlefair, P. 2002. The London heat island: Results from summertime monitoring. Building Services Engineering Research and Technology 23, 97-106. doi:10.1191/0143624402bto310a

Young, D.T., Chapman, L., Bland, S. C., Muller, C. L. 2012. Assessing a 'low-cost' wireless temperature sensor for HiTemp. $8^{\text {th }}$ International Conference on Urban Climate, $6^{\text {th }}-10^{\text {th }}$ August 2012, Dublin, Ireland. 